\title{
A SYMMETRIC ERROR ESTIMATE FOR GALERKIN APPROXIMATIONS OF TIME-DEPENDENT NAVIER-STOKES EQUATIONS IN TWO DIMENSIONS
}

\author{
TODD F. DUPONT AND ITIR MOGULTAY
}

\begin{abstract}
A symmetric error estimate for Galerkin approximations of solutions of the Navier-Stokes equations in two space dimensions plus time is given. The finite-dimensional function spaces are taken to be divergence-free, and time is left continuous. The estimate is similar to known results for scalar parabolic equations. An application of the result is given for mixed method formulations. A short discussion of examples is included. Finally, there are some remarks about a partial extension to three space dimensions.
\end{abstract}

\section{INTRODUCTION}

A symmetric error estimate for a numerical method is a statement that, in a certain norm, the error for the approximation produced by that method is best possible, up to a constant factor; i.e.,

$$
\| \text { error }\|\leq C\| \text { best approximation error } \| \text {. }
$$

For second-order elliptic problems, the basic error estimate for the Galerkin approximations is a symmetric estimate in the $H^{1}$-norm. Symmetric error estimates are called quasi-optimal by some researchers. Some symmetric error estimates for parabolic equations can be found in [3, 4, 1, 5]. In [2] an error estimate is given for the Stokes equation (see section 12.3), which is symmetric if one restricts to divergence-free spaces.

In this paper, we examine Galerkin approximations of solutions of the timedependent two-dimensional Navier-Stokes equations on a bounded domain with zero boundary conditions. We consider the case in which the approximate solution is required to be divergence free. We prove stability for the Galerkin approximation on a finite time interval in a norm that is then used in the symmetric error estimate. The symmetric estimates in this paper grew from the analysis in $[7$ in which almost symmetric estimates were produced for a model of sea breezes.

In section 2, we introduce the boundary value problem and define some notation. In section 3, we define the Galerkin approximation and give a bound for it. In section 4 , we state and prove the symmetric error estimate. In section 5 , we show that the estimate applies to certain mixed method approximations of the same

Received by the editor July 30, 2007 and, in revised form, November 9, 2008.

2000 Mathematics Subject Classification. Primary 65M12; Secondary 65M60.

Key words and phrases. Error estimates, Navier-Stokes equations, Galerkin approximation, quasi-optimal.

The work of the authors was supported in part by the ASC Flash Center at the University of Chicago which is funded by the U. S. Department of Energy under contract B523820.

(C)2009 American Mathematical Society 
problem, and we give an example of a family of spaces which could be used. In section 6 we consider the problem in three space dimensions and give an almostsymmetric error estimate.

\section{The PROBLEM AND NOTATION}

We are interested in approximating smooth solutions of the Navier-Stokes equations. Let $\Omega$ be a bounded domain in $\mathbb{R}^{2}$. For some $t_{\text {final }}>0$, let $J=\left(0, t_{\text {final }}\right)$, and take $Q=\Omega \times J$. Suppose that $\bar{u}: \bar{Q} \rightarrow \mathbb{R}^{2}, p: \bar{Q} \rightarrow \mathbb{R}$ are smooth functions such that on $Q$,

$$
\begin{aligned}
\bar{u}_{t}+\bar{u} \cdot \nabla \bar{u}+\frac{1}{\rho} \nabla p-K \triangle \bar{u} & =F, \\
\nabla \cdot \bar{u} & =0,
\end{aligned}
$$

where $\nabla$ is the spatial gradient operator, $\triangle$ is the Laplacian, and $F$ is a function in $L^{2}(Q)^{2}$. Here, $\rho$ and $K$ are positive constants, the density and kinematic viscosity, respectively. We suppose that $\bar{u}$ vanishes on the boundary of $\Omega$.

Let $\mathcal{X}$ be a norm space with norm $\|\cdot\|_{\mathcal{X}}$ and suppose that $f: J \rightarrow \mathcal{X}$. For sufficiently nice functions $f$, define for $1 \leq p \leq \infty$,

$$
\|f\|_{L^{p}(\mathcal{X})}=\|\| f(\cdot)\left\|_{\mathcal{X}}\right\|_{L^{p}(J)} .
$$

Let $(\cdot, \cdot)_{\Omega}$ denote the $L^{2}(\Omega)$ inner product. We use this notation also for the inner product on $L^{2}(\Omega)^{2}$. For vector-valued functions, $\varphi,\|\varphi\|_{L^{p}(\Omega)}$ denotes the $L^{p}(\Omega)$ norm of the Euclidean norm of $\varphi$. If $\varphi$ is vector-valued, the norm of its gradient, $|\nabla \varphi|$, is just the Euclidean norm of its components considered as a vector. For nonnegative integers $k$, we use the notation $\|f\|_{k}=\|f\|_{H^{k}(\Omega)}$, and we use the same notation for scalar or vector-valued functions. For $k=0$, we use $\|\cdot\|$. For $\mathcal{M}$ a subspace of $H_{0}^{1}(\Omega)^{2}$ and $f \in L^{2}(\Omega)^{2}$, we define the following semi-norm:

$$
\|f\|_{H_{\mathcal{M}}^{-1}}=\sup _{0 \neq \sigma \in \mathcal{M}} \frac{(f, \sigma)_{\Omega}}{\|\nabla \sigma\|} .
$$

In the case $\mathcal{M}=H_{0}^{1}(\Omega)^{2}$ we use $\|f\|_{H^{-1}}$.

A norm that plays a central role here is defined for (sufficiently nice) functions $f$ mapping $Q$ into $\mathbb{R}^{2}$ by

$$
\|f\|^{2}=\|f\|_{L^{\infty}\left(L^{2}(\Omega)\right)}^{2}+\|f\|_{L^{2}\left(H_{0}^{1}(\Omega)\right)}^{2}+\left\|f_{t}\right\|_{L^{2}\left(H_{\mathcal{M}}^{-1}\right)}^{2} .
$$

This norm depends on $\mathcal{M}$ of course.

\section{Galerkin approximation And a Bound}

Let

$$
\begin{aligned}
a(w, v) & =K \int_{\Omega} \nabla w \nabla v, \\
c(w ; s, v) & =\int_{\Omega}(w \cdot \nabla s) \cdot v,
\end{aligned}
$$

and note that for divergence-free functions $\phi \in H_{0}^{1}(\Omega)^{2}$,

$$
\left(\bar{u}_{t}, \phi\right)_{\Omega}+c(\bar{u} ; \bar{u}, \phi)+a(\bar{u}, \phi)=(F, \phi)_{\Omega}, \quad t \in J .
$$


Let $\mathcal{M}$ be a finite-dimensional subspace of the set of divergence-free functions in $H_{0}^{1}(\Omega)^{2}$. Define a function $\bar{U}$ mapping $\bar{J}$ into $\mathcal{M}$ by

$$
\begin{aligned}
& (\bar{u}(0)-\bar{U}(0), \phi)_{\Omega}=0, \quad \phi \in \mathcal{M}, \\
& \left(\bar{U}_{t}, \phi\right)_{\Omega}+c(\bar{U} ; \bar{U}, \phi)+a(\bar{U}, \phi)=(F, \phi)_{\Omega}, \quad \phi \in \mathcal{M}, \quad t \in J,
\end{aligned}
$$

where we identify $\bar{U}(t)$ with $\bar{U}(\cdot, \cdot, t)$. If $n$ is the dimension of $\mathcal{M}$, then (3.3) gives a system of $n$ ordinary differential equations that define the evolution of $\bar{U}$. The condition (3.2) defines $\bar{U}(0)$ as the $L^{2}(\Omega)$ projection of $\bar{u}(0)$ into $\mathcal{M}$. The existence of $\bar{U}$ on the entire interval follows if $\bar{U}$ is bounded on the interval where it is defined. We give such a bound here.

Theorem 3.1. There is a constant $C$ that depends on $K,\|\bar{u}(0)\|,\|F\|_{L^{2}\left(H_{\mathcal{M}}^{-1}\right)}$, and $\Omega$, such that

$$
\|\bar{U}\| \leq C .
$$

Proof. If for each $t$ we replace $\phi$ in (3.3) by $\bar{U}(t)$, we get that

$$
\frac{1}{2} \frac{d}{d t}\|\bar{U}\|^{2}+K\|\nabla \bar{U}\|^{2}=(F, \bar{U}) \leq\|F\|_{H_{\mathcal{M}}^{-1}}\|\nabla \bar{U}\| \leq \frac{1}{2} K\|\nabla \bar{U}\|^{2}+\frac{1}{2 K}\|F\|_{H_{\mathcal{M}}^{-1}}^{2} .
$$

From this, it follows that for each $t \in J$,

$$
\|\bar{U}(t)\|^{2}+K \int_{0}^{t}\|\nabla \bar{U}\|^{2} \leq \frac{1}{K} \int_{0}^{t}\|F\|_{H_{\mathcal{M}}^{-1}}^{2}+\|\bar{u}(0)\|^{2},
$$

where we used $\|\bar{U}(0)\| \leq\|\bar{u}(0)\|$. Thus

$$
\|\bar{U}\|_{L^{\infty}\left(L^{2}(\Omega)\right)}^{2}+\|\bar{U}\|_{L^{2}\left(H^{1}(\Omega)\right)}^{2} \leq C,
$$

where $C$ depends on $K,\|\bar{u}(0)\|,\|F\|_{L^{2}\left(H_{M}^{-1}\right)}$, and $\Omega$.

Take $\phi \in \mathcal{M}$ such that $\|\nabla \phi\|=1$ and $\left\|\bar{U}_{t}\right\|_{H_{\mathcal{M}}^{-1}}=\left(\bar{U}_{t}, \phi\right)_{\Omega}$. Then

$$
\begin{aligned}
\left\|\bar{U}_{t}\right\|_{H_{\mathcal{M}}^{-1}} & =-(\bar{U} \cdot \nabla \bar{U}, \phi)_{\Omega}-K(\nabla \bar{U}, \nabla \phi)_{\Omega}+(F, \phi)_{\Omega} \\
& \leq\|\bar{U}\|_{L^{4}(\Omega)}^{2}+K\|\nabla \bar{U}\|+\|F\|_{H_{\mathcal{M}}^{-1}} \\
& \leq\|\bar{U}\|\|\nabla \bar{U}\|+K\|\nabla \bar{U}\|+\|F\|_{H_{\mathcal{M}}^{-1}}
\end{aligned}
$$

where we used Lemma A.1 and then Lemma A.2, It follows that $\left\|\bar{U}_{t}\right\|_{H_{\mathcal{M}}^{-1}}$ is in $L^{2}(J)$ in time with a bound that depends only on $\|\bar{u}(0)\|, K$, and $\|F\|_{L^{2}\left(H_{M}^{-1}\right)}$. Hence $\|\bar{U}\|$ is bounded.

Remark. If we replace $H_{\mathcal{M}}^{-1}$ by $H^{-1}$ in the norm on $F$, we get (3.4) with $C$ that is independent of $\mathcal{M}$. This bound is used in the next section. Also note that the same bound holds for the true solution $\bar{u}$.

\section{Symmetric ERror estimate}

Take $\bar{W}$ to be any smooth function from $\bar{J}$ into $\mathcal{M}$. Our claim is that if $\bar{W}$ is close to $\bar{u}$, then $\bar{U}$ is also close to $\bar{u}$. That is, if the space $\mathcal{M}$ is sufficiently rich that we can approximate the solution of the Navier-Stokes equations well, then the Galerkin solution does a good job of approximating it. 
Theorem 4.1. Suppose that $B>0$. Then there is a constant $C$ that depends on $B, K$, and $Q$ such that if $\|\bar{u}(0)\| \leq B$ and $\|F\|_{L^{2}\left(H^{-1}\right)} \leq B$, then

$$
\|\bar{u}-\bar{U}\| \leq C\|\bar{u}-\bar{W}\| \text {. }
$$

Proof. We adopt the notation $\bar{U}_{1}=\bar{u}, \bar{U}_{2}=\bar{U}, \bar{U}_{3}=\bar{W}$, and $\bar{U}^{i j}=\bar{U}_{i}-\bar{U}_{j}$ to shorten the formulas. We prove that $\left\|\bar{U}^{23}\right\| \leq C\left\|\bar{U}^{13}\right\|$. The triangle inequality then gives the conclusion with $C$ replaced by $C+1$, since $\bar{u}-\bar{U}=\bar{U}^{12}=\bar{U}^{13}-\bar{U}^{23}$.

Using (3.11) and (3.3) we see that for any $\phi \in \mathcal{M}$,

$$
\begin{aligned}
\left(\bar{U}_{t}^{23}, \phi\right)_{\Omega} & +c\left(\bar{U}_{2} ; \bar{U}^{23}, \phi\right)+a\left(\bar{U}^{23}, \phi\right) \\
& =\left(\bar{U}_{t}^{13}, \phi\right)_{\Omega}+c\left(\bar{U}_{1} ; \bar{U}_{1}, \phi\right)-c\left(\bar{U}_{2} ; \bar{U}_{3}, \phi\right)+a\left(\bar{U}^{13}, \phi\right) .
\end{aligned}
$$

Taking $\phi=\bar{U}^{23}$ and using the fact that $c\left(\bar{U}_{2} ; \bar{U}^{23}, \bar{U}^{23}\right)=0$ gives

$$
\begin{aligned}
\frac{1}{2} \frac{d}{d t}\left\|\bar{U}^{23}\right\|^{2}+K\left\|\nabla \bar{U}^{23}\right\|^{2}= & \left(\bar{U}_{t}^{13}, \bar{U}^{23}\right)_{\Omega}+\left(\bar{U}_{1} \cdot \nabla \bar{U}_{1}-\bar{U}_{2} \cdot \nabla \bar{U}_{3}, \bar{U}^{23}\right)_{\Omega} \\
& +K\left(\nabla \bar{U}^{13}, \nabla \bar{U}^{23}\right)_{\Omega} .
\end{aligned}
$$

Note that since $\bar{U}_{1}$ and $\bar{U}_{2}$ are divergence-free,

$$
\left|\left(\bar{U}_{1} \cdot \nabla \bar{U}_{1}-\bar{U}_{2} \cdot \nabla \bar{U}_{3}, \bar{U}^{23}\right)_{\Omega}\right|=\left|\left(\bar{U}_{1} \bar{U}_{1}^{T}-\bar{U}_{2} \bar{U}_{3}^{T}, \nabla \bar{U}^{23}\right)_{\Omega}\right| .
$$

Then writing $\bar{U}_{1} \bar{U}_{1}{ }^{T}-\bar{U}_{2} \bar{U}_{3}{ }^{T}=\bar{U}_{1}\left(\bar{U}^{13}-\bar{U}^{23}\right)^{T}+\bar{U}_{2} \bar{U}^{13^{T}}$ and applying Cauchy's inequality gives that

$$
\begin{gathered}
\left|\left(\bar{U}_{1} \cdot \nabla \bar{U}_{1}-\bar{U}_{2} \cdot \nabla \bar{U}_{3}, \bar{U}^{23}\right)_{\Omega}\right| \leq\left(\left\|\bar{U}_{1} \bar{U}^{13^{T}}\right\|+\left\|\bar{U}_{1} \bar{U}^{23^{T}}\right\|+\left\|\bar{U}_{2} \bar{U}^{13^{T}}\right\|\right)\left\|\nabla \bar{U}^{23}\right\| \\
\leq \frac{4}{K}\left(\left\|\bar{U}^{13} \bar{U}_{1}^{T}\right\|^{2}+\left\|\bar{U}^{23} \bar{U}_{1}^{T}\right\|^{2}+\left\|\bar{U}_{2} \bar{U}^{13^{T}}\right\|^{2}\right)+\frac{K}{5}\left\|\nabla \bar{U}^{23}\right\|^{2}
\end{gathered}
$$

Each of the terms involving the $L^{2}$-norm of the product of two functions will be bounded using Lemma A.2 in the same way. We illustrate that with the middle term because it needs a little more care:

$$
\begin{aligned}
\left\|\bar{U}^{23} \bar{U}_{1}^{T}\right\|^{2} & \leq\left\|\bar{U}^{23}\right\|_{L^{4}(\Omega)}^{2}\left\|\bar{U}_{1}\right\|_{L^{4}(\Omega)}^{2} \\
& \leq\left\|\bar{U}^{23}\right\|\left\|\nabla \bar{U}^{23}\right\|\left\|\bar{U}_{1}\right\|\left\|\nabla \bar{U}_{1}\right\| \\
& \leq C\left\|\nabla \bar{U}_{1}\right\|^{2}\left\|\bar{U}^{23}\right\|^{2}+\frac{K}{4} \frac{K}{5}\left\|\nabla \bar{U}^{23}\right\|^{2},
\end{aligned}
$$

where the $C$ depends on $K$ and an upper bound for $\|\bar{u}\|_{L^{\infty}\left(L^{2}(\Omega)\right)}$. The bounds

$$
\begin{aligned}
\left|\left(\bar{U}_{t}^{13}, \bar{U}^{23}\right)_{\Omega}\right| & \leq C\left\|\bar{U}_{t}^{13}\right\|_{H_{\mathcal{M}}^{-1}}^{2}+\frac{K}{5}\left\|\nabla \bar{U}^{23}\right\|^{2}, \\
\left|\left(K \nabla \bar{U}^{13}, \nabla \bar{U}^{23}\right)_{\Omega}\right| & \leq C\left\|\nabla \bar{U}^{13}\right\|^{2}+\frac{K}{5}\left\|\nabla \bar{U}^{23}\right\|^{2}
\end{aligned}
$$

then imply that

$$
\begin{aligned}
\frac{d}{d t}\left\|\bar{U}^{23}\right\|^{2}+\frac{K}{5}\left\|\nabla \bar{U}^{23}\right\|^{2} \leq & C\left[\left\|\bar{U}_{t}^{13}\right\|_{H_{\mathcal{M}}^{-1}}^{2}+\left\|\nabla \bar{U}_{1}\right\|^{2}\left\|\bar{U}^{23}\right\|^{2}\right. \\
& \left.+\left(1+\left\|\bar{U}_{2}\right\|^{2}\right)\left\|\bar{U}^{13}\right\|_{1}^{2}+\left(1+\left\|\bar{U}_{2}\right\|_{1}^{2}\right)\left\|\bar{U}^{13}\right\|^{2}\right] .
\end{aligned}
$$


Note that (3.2) says that, at $t=0, \bar{u}-\bar{U}=\bar{U}^{12}=\bar{U}^{13}-\bar{U}^{23}$ is orthogonal to $\mathcal{M}$ (in particular $\bar{U}^{23}$ ), which gives $\left\|\bar{U}^{23}(0)\right\| \leq\left\|\bar{U}^{13}(0)\right\|$. Now, Gronwall's inequality yields

$$
\left\|\bar{U}^{23}\right\|_{L^{\infty}\left(L^{2}(\Omega)\right)}^{2}+\left\|\bar{U}^{23}\right\|_{L^{2}\left(H^{1}(\Omega)\right)}^{2} \leq C\left\|\bar{U}^{13}\right\|^{2} .
$$

In applying Gronwall's inequality we used that for $t \in J$,

$$
\int_{0}^{t}\left(\left(1+\left\|\bar{U}_{2}\right\|^{2}\right)\left\|\bar{U}^{13}\right\|_{1}^{2}+\left(1+\left\|\bar{U}_{2}\right\|_{1}^{2}\right)\left\|\bar{U}^{13}\right\|^{2}\right) \leq C\left\|\bar{U}^{13}\right\|^{2}
$$

and the fact that $\left\|\nabla \bar{U}_{1}(\cdot, t)\right\|^{2}$ is in $L^{1}(J)$.

To complete the proof we need only bound the negative index semi-norm of $\bar{U}_{t}^{23}$. Take $\phi \in \mathcal{M}$ such that $\|\nabla \phi\|=1$ and $\left\|\bar{U}_{t}^{23}\right\|_{H_{\mathcal{M}}^{-1}}=\left(\bar{U}_{t}^{23}, \phi\right)_{\Omega}$. From (4.1), we have

$$
\begin{aligned}
\left(\bar{U}_{t}^{23}, \phi\right)_{\Omega}= & \left(\bar{U}_{t}^{13}, \phi\right)_{\Omega}+a\left(\bar{U}^{13}-\bar{U}^{23}, \phi\right)+\left(\bar{U}_{1} \cdot \nabla \bar{U}_{1}-\bar{U}_{2} \cdot \nabla \bar{U}_{2}, \phi\right)_{\Omega} \\
= & \left(\bar{U}_{t}^{13}, \phi\right)_{\Omega}+a\left(\bar{U}^{13}-\bar{U}^{23}, \phi\right) \\
& \quad+\left(\left(\bar{U}^{23}-\bar{U}^{13}\right) \bar{U}_{1}^{T}+\bar{U}_{2}\left(\bar{U}^{23}-\bar{U}^{13}\right)^{T}, \nabla \phi\right)_{\Omega} .
\end{aligned}
$$

Thus

$$
\begin{aligned}
\left\|\bar{U}_{t}^{23}\right\|_{H_{\mathcal{M}}^{-1}} & \leq C\left(\left\|\bar{U}_{t}^{13}\right\|_{H_{\mathcal{M}}^{-1}}+\left\|\bar{U}^{13}\right\|_{1}+\left\|\bar{U}^{23}\right\|_{1}\right) \\
& +\left(\left(\bar{U}^{23}-\bar{U}^{13}\right) \bar{U}_{1}^{T}+\bar{U}_{2}\left(\bar{U}^{23}-\bar{U}^{13}\right)^{T}, \nabla \phi\right)_{\Omega} .
\end{aligned}
$$

The terms in the big parentheses are bounded in $L^{2}(J)$ by $C\left\|\bar{U}^{13}\right\|$. The last term on the right hand side is treated using Lemmas A.1 and A.2. We illustrate using only the $\bar{U}_{2} \bar{U}^{23}$ part. As above,

$$
\begin{aligned}
\left|\left(\bar{U}_{2} \bar{U}^{23^{T}}, \nabla \phi\right)_{\Omega}\right| & \leq\left\|\bar{U}_{2}\right\|_{L^{4}(\Omega)}\left\|\bar{U}^{23}\right\|_{L^{4}(\Omega)} \\
& \leq\left(\left\|\bar{U}_{2}\right\|\left\|\nabla \bar{U}_{2}\right\|\left\|\bar{U}^{23}\right\|\left\|\nabla \bar{U}^{23}\right\|\right)^{\frac{1}{2}} .
\end{aligned}
$$

If $\mu(t)=\left(\left\|\bar{U}_{2}\right\|\left\|\nabla \bar{U}_{2}\right\|\left\|\bar{U}^{23}\right\|\left\|\nabla \bar{U}^{23}\right\|\right)^{\frac{1}{2}}$, we have, using (3.4) and (4.2),

$$
\begin{aligned}
\int_{J} \mu^{2} d t & \leq \frac{1}{2} \int_{J}\left(\left\|\bar{U}_{2}\right\|_{1}^{2}\left\|\bar{U}^{23}\right\|^{2}+\left\|\bar{U}_{2}\right\|^{2}\left\|\bar{U}^{23}\right\|_{1}^{2}\right) \\
& \leq C\left\|\bar{U}^{13}\right\| .
\end{aligned}
$$

Thus we see that $\left\|\bar{U}_{t}^{23}\right\|_{L^{2}\left(H_{\mathcal{M}}^{-1}\right)} \leq C\left\|\bar{U}^{13}\right\|$, and therefore $\left\|\bar{U}^{23}\right\| \leq C\left\|\bar{U}^{13}\right\|$.

For the symmetric error estimates for the nonlinear scalar parabolic equations derived in [4] it was found necessary to impose qualitative bounds on $W$. Here, such a constraint was not needed, because the nonlinearity is a lower-order term.

\section{Application to mixed methods}

Suppose that $V_{h} \subset H_{0}^{1}(\Omega)^{2}$ and $\Pi_{h} \subset L^{2}(\Omega)$ are finite-dimensional spaces. Define

$$
b(w, z)=-\int_{\Omega}(\operatorname{div} w) z .
$$


Then a mixed formulation approximation can be defined by requiring that for each $t \in J$

$$
\begin{aligned}
& \left(\bar{U}_{t}, v\right)_{\Omega}+c(\bar{U} ; \bar{U}, v)+a(\bar{U}, v)+b(v, P)=(F, v)_{\Omega}, \quad v \in V_{h}, \\
& b(\bar{U}, q)=0, \quad q \in \Pi_{h},
\end{aligned}
$$

where $\bar{U}: \bar{J} \rightarrow V_{h}$ and $P: \bar{J} \rightarrow \Pi_{h}$. The initial conditions $\bar{U}(0)$ and $P(0)$ are taken such that

$$
\begin{aligned}
& (\bar{U}(0)-\bar{u}(0), v)_{\Omega}+b(v, P(0))=0, \quad v \in V_{h}, \\
& b(\bar{U}(0), q)=0, \quad q \in \Pi_{h} .
\end{aligned}
$$

Now suppose that $V_{h}$ and $\Pi_{h}$ have been chosen so that the set

$$
\mathcal{M}=\left\{w \in V_{h}: b(w, q)=0 \text { for all } q \in \Pi_{h}\right\}
$$

contains only divergence-free functions. Not all reasonable mixed method spaces will have this property, but some do, for example, $\Pi_{h}=d i v V_{h}$. With this assumption, $\bar{U}: \bar{J} \rightarrow \mathcal{M}$, and, on $J, \bar{U}$ satisfies

$$
\left(\bar{U}_{t}, v\right)_{\Omega}+c(\bar{U} ; \bar{U}, v)+a(\bar{U}, v)=(F, v)_{\Omega}, \quad v \in \mathcal{M} .
$$

Also the initial value $\bar{U}(0)$ is in $\mathcal{M}$ and satisfies

$$
(\bar{U}(0)-\bar{u}(0), v)_{\Omega}=0, \quad v \in \mathcal{M} .
$$

Hence the symmetric error estimate applies to $\bar{U}$.

Examples. Define $V_{h}=V_{h}^{x} \times V_{h}^{y}$ by defining the spaces for the $x$ - and $y$-velocities and take $\Pi_{h}=\operatorname{div} V_{h}$. Then the symmetric error estimate applies, but the space $\mathcal{M}$ may or may not be able to approximate the solution. We will briefly discuss some examples in the case $\Omega=(0,1) \times(0,1)$. For simplicity we will restrict attention to a uniform mesh of size $h=1 / N$ in the $x$ - and $y$-variables.

Since $V_{h}^{x}$ and $V_{h}^{y}$ must both be subspaces of $H_{0}^{1}$, the simplest space would be to take both $V_{h}^{x}$ and $V_{h}^{y}$ to be tensor products of continuous piecewise linears vanishing on the boundary. It is easy to see that the dimension of the divergence-free subspace of $V_{h}$ is the dimension of $\partial_{x} V_{h}^{x} \cap \partial_{y} V_{h}^{y}$. In this case the dimension of the intersection is zero; i.e., the only function in $\mathcal{M}$ vanishes identically. Clearly this choice of $V_{h}$ is not a good one.

Next take $\mathcal{N} \subset H_{0}^{2}(\Omega)$ to be the tensor product of $C^{1}$ piecewise quadratics on $[0,1]$ which vanish to second order at the ends. Then set $V_{h}^{x}=\partial_{y} \mathcal{N}$ and $V_{h}^{y}=\partial_{x} \mathcal{N}$. In this case $\mathcal{M}=\operatorname{curl} \mathcal{N}$, and we get that the scheme is first-order correct in the $\|\cdot\|$ norm. (See 2], section 12.4, for details.)

The above example is easily extended to a family of schemes that are of order $k>1$; one starts with the space $\mathcal{N}$ that is the tensor product of $C^{1}$ piecewise polynomials of degree $k+1$.

\section{Three SPACE Dimensions}

In this section we look at extending the 2-d results to 3-d. It might seem that if the assumptions on $\bar{u}$ were strong enough the theorems would be unchanged. However, it is the stability of the Galerkin process that governs what can be done; as always, it is the stability of the numerical scheme that is crucial for convergence. The analysis we have used gives an error estimate that is almost symmetric, because 
we have only weak control on $\bar{U}$. The norm that appears in this section was used in [7] in a similar way.

First we give a stability result. To this end we introduce the notation

$$
\|f\|_{p}^{2}=\|f\|_{L^{\infty}\left(L^{2}(\Omega)\right)}^{2}+\|f\|_{L^{2}\left(H_{0}^{1}(\Omega)\right)}^{2}+\left\|f_{t}\right\|_{L^{p}\left(H_{\mathcal{M}}^{-1}\right)}^{2},
$$

for $1 \leq p \leq \infty$. When $p=2$ this is the norm introduced in section 2 , and in that case the subscript on the norm is omitted.

Theorem 6.1. There is a constant $C$ that depends on $K,\|\bar{u}(0)\|,\|F\|_{L^{2}\left(H_{\mathcal{M}}^{-1}\right)}$, and $\Omega$, such that

$$
\|\bar{U}\|_{\frac{4}{3}} \leq C .
$$

Proof. The energy estimate (3.4) was derived without any constraint on the dimension, so $\bar{U}$ is bounded in $L^{\infty}\left(L^{2}(\Omega)\right)$ and $L^{2}\left(H^{1}(\Omega)\right)$. When we try to get a bound on the $H_{\mathcal{M}}^{-1}(\Omega)$ norm of the time derivative, the only troublesome part is the nonlinear term. We get at each $t$,

$$
\left\|\bar{U}_{t}\right\|_{H_{\mathcal{M}}^{-1}(\Omega)} \leq\|\bar{U}\|_{L^{4}(\Omega)}^{2}+\left[K\|\nabla \bar{U}\|^{2}+\|F\|_{H_{\mathcal{M}}^{-1}}^{2}\right]
$$

where the terms in brackets are treated as in 2-d. Next note that

$$
\int_{\Omega} \varphi^{4} \leq\|\varphi\|\|\varphi\|_{L^{6}(\Omega)}^{3}
$$

By Sobolev's embedding theorem in three dimensions, $\|\varphi\|_{L^{6}(\Omega)} \leq C\|\varphi\|_{H^{1}(\Omega)}$. Hence

$$
\|\bar{U}\|_{L^{4}(\Omega)} \leq C\|\bar{U}\|^{\frac{1}{4}}\|\nabla \bar{U}\|^{\frac{3}{4}} .
$$

The bounds we have on the $L^{\infty}(J)$-norm of $\|\bar{U}\|$ and the $L^{2}(J)$-norm of $\|\nabla \bar{U}\|$ imply that $\|\bar{U}\|_{L^{4}(\Omega)}^{2}$ is in $L^{\frac{4}{3}}(J)$. This gives the claimed result.

In order to get an almost-symmetric error estimate in the three-space-dimensional context we need to restrict the function $W$ to be "nice". It seems reasonable that if $\bar{u}$ is bounded on $Q$, then there is a function in the space $\mathcal{M}$ that matches it well but is also well behaved, in the sense that its max norm is no more than (say) twice that of $\bar{u}$. This is similar to the approach that was used in [4 for nonlinear parabolic equations.

Theorem 6.2. Suppose that $B$ and $D$ are positive. Then there is a constant $C$ that depends on $B, D, K$, and $Q$ such that if $\|\bar{u}\|_{L^{\infty}(Q)} \leq B,\|F\|_{L^{2}\left(H^{-1}\right)} \leq B$, and $\|\bar{W}\|_{L^{\infty}(Q)} \leq D$, then

$$
\|\bar{u}-\bar{U}\|_{\frac{4}{3}} \leq C\|\bar{u}-\bar{W}\| .
$$

Proof. To get the analog of (4.2) the nonlinear term is treated differently. Write $\bar{U}_{1} \bar{U}_{1}^{T}-\bar{U}_{2} \bar{U}_{3}^{T}=\bar{U}_{1} \bar{U}^{13^{T}}+\left(\bar{U}^{13}-\bar{U}^{23}\right) \bar{U}_{3}^{T}$ and use the assumed bounds on $\bar{U}_{1}$ and $\bar{U}_{3}$ to get

$$
\left|\left(\bar{U}_{1} \cdot \nabla \bar{U}_{1}-\bar{U}_{2} \cdot \nabla \bar{U}_{3}, \bar{U}^{23}\right)_{\Omega}\right| \leq C\left(\left\|\bar{U}^{13}\right\|^{2}+\left\|\bar{U}^{23}\right\|^{2}\right)+\frac{K}{4}\left\|\nabla \bar{U}^{23}\right\|^{2} .
$$

This gives, somewhat more simply than before, that

$$
\left\|\bar{U}^{23}\right\|_{L^{\infty}\left(L^{2}(\Omega)\right)}^{2}+\left\|\bar{U}^{23}\right\|_{L^{2}\left(H^{1}(\Omega)\right)}^{2} \leq C\left\|\bar{U}^{13}\right\|^{2} .
$$

In getting a bound on the time derivative part of the norm we proceed as in the last part of the proof of Theorem 4.1. The term that is problematic is 
$\left\|\bar{U}_{2}\right\|_{L^{4}(\Omega)}\left\|\bar{U}^{23}\right\|_{L^{4}(\Omega)}$. This is treated as in (6.1) to see that it is in $L^{\frac{4}{3}}(J)$, and that completes the proof.

One could imagine a different approach than presented here. If the space $\mathcal{M}$ were taken from a regular family of spaces such that the Galerkin solution $\bar{U}$ was always well behaved (bounded), then we would have more control of the stability of the numerical scheme. We have not done this, since our goal in this paper was to give estimates that allowed all spaces $\mathcal{M}$ that could approximate the solution well.

\section{Appendix A. Two Bounds}

The following two lemmas were useful in the proofs of the preceding sections.

Lemma A.1. Suppose that $\varphi, \psi$, and $\eta$ are in $H_{0}^{1}(\Omega)^{2}$ and that $\varphi$ is divergence-free. Then

$$
\left|(\varphi \cdot \nabla \psi, \eta)_{\Omega}\right| \leq\|\varphi\|_{L^{4}(\Omega)}\|\psi\|_{L^{4}(\Omega)}\|\nabla \eta\| .
$$

Proof. Let $\varphi=\left(\varphi_{1}, \varphi_{2}\right), \psi=\left(\psi_{1}, \psi_{2}\right)$, and $\eta=\left(\eta_{1}, \eta_{2}\right)$. Then

$$
\begin{aligned}
\left|(\varphi \cdot \nabla \psi, \eta)_{\Omega}\right| & =\left|\left(\varphi_{1} \psi_{1, x}+\varphi_{2} \psi_{1, y}, \eta_{1}\right)_{\Omega}+\left(\varphi_{1} \psi_{2, x}+\varphi_{2} \psi_{2, y}, \eta_{2}\right)_{\Omega}\right| \\
& =\left|\left(\partial_{x}\left(\varphi_{1} \psi_{1}\right)+\partial_{y}\left(\varphi_{2} \psi_{1}\right), \eta_{1}\right)_{\Omega}+\left(\partial_{x}\left(\varphi_{1} \psi_{2}\right)+\partial_{y}\left(\varphi_{2} \psi_{2}\right), \eta_{2}\right)_{\Omega}\right| \\
& =\left|\int_{\Omega}\left(\varphi_{1} \psi_{1} \eta_{1, x}+\varphi_{2} \psi_{1} \eta_{1, y}+\varphi_{1} \psi_{2} \eta_{2, x}+\varphi_{2} \psi_{2} \eta_{2, y}\right)\right| \\
& \leq \int_{\Omega}\left(\left(\varphi_{1}^{2} \psi_{1}^{2}+\varphi_{2}^{2} \psi_{1}^{2}+\varphi_{1}^{2} \psi_{2}^{2}+\varphi_{2}^{2} \psi_{2}^{2}\right)^{\frac{1}{2}}\left(\eta_{1, x}^{2}+\eta_{1, y}^{2}+\eta_{2, x}^{2}+\eta_{2, y}^{2}\right)^{\frac{1}{2}}\right) \\
& =\int_{\Omega}(|\varphi\|\psi\| \nabla \eta|) \\
& \leq\|\varphi\|_{L^{4}(\Omega)}\|\psi\|_{L^{4}(\Omega)}\|\nabla \eta\| .
\end{aligned}
$$

The following result can be found in [6], page 70, Lemme 6.2 and [8], page 291, Lemma 3.3; the constants are a little different.

Lemma A.2. If $v \in H_{0}^{1}(\Omega)$ where $\Omega$ is a bounded domain in $R^{2}$, then

$$
\|v\|_{L^{4}(\Omega)}^{4} \leq\|v\|^{2}\left\|v_{x}\right\|\left\|v_{y}\right\| \leq \frac{1}{2}\|v\|^{2}\|\nabla v\|^{2} .
$$

Proof. Without loss of generality, we can assume that $v \in C_{o}^{\infty}(\Omega)$ and is extended by zero to $\mathbb{R}^{2}$. For smooth $g$ with compact support on $\mathbb{R}$ it is elementary that

$$
\max g^{2} \leq \int\left|g g^{\prime}\right| d x
$$

Now,

$$
\begin{aligned}
\iint v^{4} d x d y & \leq \int\left[\max _{x} v^{2}(x, y) \int v^{2} d x\right] d y \\
& \leq\left(\max _{y} \int v^{2}(x, y) d x\right)\left(\int \max _{x} v^{2}(x, y) d y\right) \\
& \leq\left(\int \max _{y} v^{2}(x, y) d x\right)\left(\int \max _{x} v^{2}(x, y) d y\right) .
\end{aligned}
$$


Hence

$$
\begin{aligned}
& \|v\|_{L^{4}(\Omega)}^{4} \leq\left(\iint\left|v v_{y}\right| d y d x\right)\left(\iint\left|v v_{x}\right| d x d y\right) \leq\|v\|^{2}\left\|v_{y}\right\|\left\|v_{x}\right\| \\
& \leq\|v\|^{2} \frac{1}{2}\left(\left\|v_{y}\right\|^{2}+\left\|v_{x}\right\|^{2}\right)=\frac{1}{2}\|v\|^{2}\|\nabla v\|^{2}
\end{aligned}
$$

It is an easy exercise to see that Lemma A.2 holds for functions in $H_{0}^{1}(\Omega)^{2}$, with the same constants.

\section{REFERENCES}

1. R. E. Bank and R. F. Santos, Analysis of some moving space-time finite element methods, SIAM J. Numer. Anal. 30 (1993), 1-18. MR1202654 (93m:65141)

2. Susanne C. Brenner and L. Ridgway Scott, The mathematical theory of finite element methods, Springer-Verlag, 2008. MR2373954 (2008m:65001)

3. Jim Douglas, Jr. and Todd Dupont, Galerkin methods for parabolic equations, SIAM J. Numer. Anal. 7 (1970), no. 4, 575-626. MR0277126 (43:2863)

4. T. F. Dupont, Mesh modification for evolution equations, Mathematics of Computation 36 (1982), 85-107. MR658215 (84g:65131)

5. T. F. Dupont and Y. Liu, Symmetric error estimates for moving mesh Galerkin methods for advection-diffusion equations, SIAM J. Numer. Anal. 40 (2002), no. 3, 914-927. MR1949398 (2004b:65147)

6. J. L. Lions, Quelques méthodes de résolution des problèmes aux limites non linéaires, Dunod, 1969. MR0259693 (41:4326)

7. I. Mogultay, T. F. Dupont, and G. Eshel, Dimension reduction applied to a model of sea breezes, Tech. report, U. of Chicago, Dept. of Computer Science, TR-2007-06.

8. R. Temam, Navier-Stokes equations, theory and numerical anaysis, North-Holland, 1984. MR.769654 (86m:76003)

Department of Computer Science, University of Chicago, 1100 East 58th Street, Chicago, Illinois 60637

E-mail address: t-dupont@uchicago.edu

Department of Mathematics, University of Chicago, 5734 S. University Avenue, Chicago, IlLinois 60637

E-mail address: imogulta@cs.uchicago.edu

Current address: Yeditepe University, Department of Mathematics, 26 Augustos Yerleskesi Kayisdagi, Caddesi 81120 Kayisdagi, Istanbul Turkey 